\title{
Editorial
}

\section{Anaplastic large cell lymphoma: what's in a name?}

Although the field of neoplastic haematopathology has never been at a loss for terminological controversy, the entity that until further notice is identified as "anaplastic large cell lymphoma" (ALCL), has provoked an exceptionally persistent confusion of tongues.

In 1985, Stein et al initiated the history of ALCL by isolating a group of tumours "comprised solely of cells showing both morphological (anaplastic features) and immunological (CD30 immunoreactivity) similarities to the neoplastic cells in Hodgkin's disease" in the lymphoma category Rappaport would have indicated as "diffuse histiocytic lymphoma". ${ }^{1}$ Because the diagnosis " $\mathrm{Ki}-1$ positive large cell lymphoma" could be established quite reproducibly, and because this malignancy seemed to feature distinctive morphological, immunophenotypical, genotypical, and clinical properties, ALCL of T or null cell phenotype was ultimately recognised by the members of the international lymphoma study group and incorporated in the REAL classification as a separate entity. ${ }^{2}$

However, with regard to none of the four fundamental aspects characterising a given malignancy do ALCLs appear a homogeneous collection.

By pure morphology, at least seven different subtypes have been recognised thus far. ${ }^{3}$ Furthermore, several cases of CD30 negative, but otherwise typical ALCL cases have been described, despite the fact that CD30 immunopositivity initially was considered as conditio sine qua non to reach the diagnosis of ALCL. In general, and in consideration of the REAL classification, only neoplasms displaying a $\mathrm{T} /$ null cell immunophenotype are included, referring CD30 positive large B cell lymphomas displaying important cellular pleomorphism to the diffuse large B cell lymphoma wastebasket. Nevertheless, several investigators have claimed that the clinicopathological characteristics of B cell ALCL on the one hand and T/null cell ALCL on the other do not differ significantly, and that therefore a distinction between both cannot be justified.

More importantly, the description of a very specific chromosomal aberration, $\mathrm{t}(2 ; 5)(\mathrm{p} 23 ; \mathrm{q} 35)$, non-randomly associated with ALCL, delineated a genotypically distinct subgroup within ALCL. Because ALCL consequently appeared heterogeneous from a genotypical and therefore presumably also from a pathogenetic point of view, the importance of recognising ALCL as a distinct lymphoma category was called into question. Although a distinction between ALCL with or without anaplastic lymphoma kinase (ALK) expression based exclusively on morphological criteria is difficult, in our own experience, ALK positive ALCLs tend to be composed of a less pleomorphic neoplastic cell population. ${ }^{4}$ Moreover, as demonstrated by the study of ten Berge and colleagues ${ }^{5}$ and by several other recent publications, ALK positive ALCLs seem to be characterised by typical clinical features, including an exceptionally favourable prognosis. ${ }^{6}$ Hence, it has been suggested that ALK positive ALCL should be differentiated from ALK negative ALCL and various terms, including "ALKoma", "ALK positive lymphoma", and "primary classical anaplastic large cell lymphoma", have been proposed to denominate the novel lymphoma subtype.

In contrast to ALKomas, which are becoming better characterised, the true nature of ALK negative ALCL remains obscure. One may wonder whether a distinction with peripheral $\mathrm{T}$ cell lymphomas, not otherwise specified, is still indicated. Although it has been confirmed that, as a whole, ALCLs carry a significantly better prognosis than other large $\mathrm{T}$ cell lymphomas, this effect may exclusively result from the excellent outcome of ALKomas, which, according to the most recent studies, make up at least $60 \%$ of ALCLs. However, only a large multicentre trial comparing the clinical properties of ALK negative ALCLs with those of peripheral $\mathrm{T}$ cell lymphomas, unspecified, can ultimately resolve this intricate matter.

In addition, it will be absolutely essential to define criteria to distinguish primary cutaneous ALCL from systemic CD30 positive neoplasms because, although immunomorphologically indistinguishable, these cutaneous lymphomas show very distinct clinical features. Even if cohesive sheets of large atypical CD30 positive lymphoid cells are discerned, the disease is often curable and, in general, the prognosis of cutaneous ALCL is only slightly worse than that of lymphomatoid papulosis. In fact, it is generally assumed that these cutaneous CD30 lymphoproliferative disorders comprise a clinicopathological spectrum, characterised by a favourable outcome. ${ }^{7}$

The study by ten Berge et al, showing overall absence of ALK expression in primary cutaneous CD30 positive lymphoproliferative disorders indicates that, despite their similarities in terms of prognosis, primary cutaneous CD30 positive lymphoproliferative disorders clearly differ from ALKomas in terms of genotype, and therefore probably represent an intrinsically different type of neoplasm.

On the other hand, systemic ALK negative ALCLs run an aggressive and often incurable clinical course, especially when the skin is involved. As yet, only a thorough clinical investigation can exclude the presence of systemic disease and guide the crucial choice between a conservative approach and the administration of a very aggressive chemotherapeutic regimen. Consequently, valid and reproducible criteria are required to discriminate between primary cutaneous ALCL and systemic ALK negative ALCL disseminating to the skin.

In summary, the recognition of a specific subtype within ALCL, characterised by expression of the ALK protein, has prompted a re-appraisal of the definition of ALCL. It is tempting to speculate that the broadened perspective that should result from a rational discussion on the subject and from further studies unravelling the true nature of these neoplasms might ultimately demonstrate that at present there are just too many different things in the name ALCL ...

CHRIS DE WOLF-PEETERS

RUTH ACHTEN

Department of Pathology, UZ KULeuven, Minderbroedersstraat 12, B-3000 Leuven, Belgium 
1 Stein H, Mason DY, Gerdes J, et al. The expression of the Hodgkin's disease associated antigen $\mathrm{Ki}-1$ in reactive and neoplastic lymphoid tissue: evidence that Reed-Sternberg cells and histiocytic malignancies are derived from activated lymphoid cells. Blood 1985;66:848-58.

2 Harris NL, Jaffe ES, Stein H, et al. A revised European-American classification of lymphoid neoplasms: a proposal from the international lymphoma study group [see comments]. Blood 1994;84:1361-92

3 Kadin ME. Anaplastic large cell lymphoma and its morphological variants. Cancer Surv 1997;30:77-86.

4 Pittaluga S, Wiodarska T, Pullford K, et al. The monoclonal antibody ALK1 identifies a distinct morphological subtype of anaplastic large cell lymphoma associated with 2p23/ALK rearrangements. Am f Pathol 1997;151:343-51.
5 Falini B, Pileri S, Zinzani PL, et al. ALK+ lymphoma: clinico-pathological findings and outcome. Blood 1999;93:2697-706.

6 ten Berge RL, Oudejans JJ, Ossenkoppele GJ, et al. ALK expression in extranodal anaplastic large cell lymphoma favours systemic disease with (primary) nodal involvement and a good prognosis and occurs before dissemination. F Clin Pathol 2000;53:445-50.

7 Paulli M, Berti E, Rosso R, et al. CD-30/Ki-1-positive lymphoproliferative disorders of the skin - clinicopathologic correlation and statistical analysis of 86 cases: a multicentric study from the European organization for research and treatment of cancer cutaneous lymphoma project group. $\mathcal{F}$ Clin Oncol 1995;13:1343-54. 